\title{
APROVECHAMIENTO DEL RETAMO ESPINOSO COMO FUENTE POTENCIAL DE AZÚCARES FERMENTABLES PARA COMBUSTIBLES DE SEGUNDA GENERACIÓN A TRAVÉS DE HIDROLISISÁCIDA
}

\author{
Use of Ulex Europaeus as a potential source of fermentable sugars for second generation \\ fuels through acid hydrolysis.
}

\author{
Barragán Sanabria Laura Valentina \\ Lbarragan6o@unisalle.edu.co
}

Méndez Torres Yeferson Stiven

Ymendez66@unisalle.edu.co

Universidad de La Salle

Ingeniería Ambiental y Sanitaria

Colombia

\section{Resumen}

El retamo espinoso (Ulex europeaus) es una especie exótica y foránea, originario de las sabanas secas del centro de Europa y se introdujo en Bogotá D.C. hacia los años 50. Al encontrar en nuestros ecosistemas condiciones excepcionales de clima y disposición de nutrientes, su capacidad de regeneración, adaptación y resistencia a condiciones extremas, facilitan su permanencia y dispersión (Ecofondo, s.f). Por sus características biológicas de baja humedad y contenido de aceite, contiene un alto potencial para su transformación en azúcares reductores y a partir de ellos la generación de biocombustibles de segunda generación; combustibles líquidos fabricados a partir de la biomasa lignocelulósica de plantas (Hackenberg, s.f). Para su aprovechamiento, se realizó un diseño experimental a nivel de laboratorio, en el cual se aplicó el proceso de hidrólisis ácida para la obtención de azúcares reductores, se utilizaron concentraciones de ácido sulfúrico ( $\mathrm{H} 2 \mathrm{SO} 4)$ al $0, \mathrm{I} \mathrm{N}, 0,3 \mathrm{~N}$ y $0,5 \mathrm{~N}$ a una temperatura de 30

${ }^{\circ} \mathrm{C}$ y $150 \mathrm{rpm}$ en un agitador orbital (IKA KS $4000 \mathrm{i}$ control) durante 24 horas con una cantidad de $5 \mathrm{~g}$ de retamo espinoso por tratamiento. Se encontró que las mejores condiciones para la hidrólisis ácida de retamo espinoso fueron agitación a $150 \mathrm{rpm}$ a una temperatura de $30^{\circ} \mathrm{C}$ y una concentración de $0, \mathrm{I} \mathrm{N}$ de ácido sulfúrico $(\mathrm{H} 2 \mathrm{SO}$ ) con una liberación de I58,7 mg de AR/g de residuo seco, por ende se concluye que la mejor concentración para desarrollar la hidrólisis ácida de retamo espinoso es ácido sulfúrico a $0.1 \mathrm{~N}$, en donde hubo una conversión de casi el $16 \%$ en azúcares reductores con respecto a los gramos de residuo seco utilizado en comparación con las otras concentraciones, en donde se obtuvo para $0,3 \mathrm{~N}$ y $0,5 \mathrm{~N}$ de ácido sulfúrico, un porcentaje de conversión del $8 \%$ y $0,5 \%$ respectivamente.

Palabras clave:

Retamo espinoso, Azucares reductores, biocombustibles, biomasa lignocelulosica, hidrolisis acida, 
En la actualidad los combustibles fósiles que son utilizados tienen una gran problemática ambiental, debido a que la combustión de estos genera contaminación atmosférica, del agua y suelo (Por el desecho de aceites quemados). Según (Hackenberg, 2018), frente a dichas problemáticas, se han evaluado alternativas innovadoras como los biocombustibles.

La necesidad de la reducción de emisiones de gases efecto invernadero han promovido la investigación de combustibles alternativos. Como respuesta a esto aparecen los combustibles de segunda generación, que son aquellos que se producen a través de residuos lignocelulósicos y presentan la oportunidad de solución más promisoria debido a la gran cantidad de biomasa residual disponible. De acuerdo con lo anterior el retamo espinoso por sus características biológicas presenta una oportunidad para su transformación a azucares reductores y un potencial frente a la generación de bioetanol.

Método

\section{RECOLECCIÓN DE MUESTRAS Y PRE - EXPERIMENTAL:}

El proceso de recolección del retamo espinoso según el Ministerio de Medio Ambiente y Desarrollo sostenible se da de la siguiente manera:

-Diagnóstico del área que se va a intervenir. Incluye una delimitación del espacio, caracterización de los matorrales retamo (datos de altura y diámetro) y un mapa de ese sector

\section{CONTROL}

Para llevar a cabo el control de las especies de Retamo espinoso, se establecen procedimientos de eliminación manual y mecánico.

- En el control de las ramificaciones leñosas se podrá utilizar machete, guadaña, motosierra o cualquier otra herramienta que facilite este procedimiento.

- Se deben dejar tocones de 20 a $30 \mathrm{~cm}$ del tronco principal del retamo, para que facilite la extracción de raíces de la planta.

- Los tocones (parte del tallo que se deja para después extraer con la raíz) y raíces deberán ser removidos utilizando herramientas como pica, barretón, azadón o cualquier otra herramienta que permita aflojar el terreno, haciendo palanca al sistema radicular en una profundidad de $20-30 \mathrm{~cm}$

- Para su intervención podrá utilizar maquinarias como el tractor o maquinarias que permitan intervenciones en grandes superficies.

- Se preparan las áreas donde se van a apilar, es decir el suelo deberá estar cubierto de plástico (calibre 6), para que no se llegue a dispersar a un sustrato donde puedagerminar.

- El apilamiento y confinamiento del material deberá realizarse solamente en las zonas habilitadas para esto, y la altura del material acumulado no podrá superar los 2 metros.

- Para evitar la dispersión de los propágalos de la especie. Se sugiere realizar acciones complementarias, como desarrollar de manera paralela al control manual y mecánico previamente descrito, la construcción de trampas de semilla, zanjas, cunetas, drenaje, interceptores de semillas y trinchos; acciones que deberán adelantarse orientadas a disminuir las vías de dispersión. 


\section{MITIGACIÓN}

-En el caso de las herramientas empleadas en la manipulación de especies de retamo, antes de que estas sean desplazadas de un lugar a otro, deberá revisarse que no tenga ninguna parte o semilla, de la especie adherida a las mismas.

-Se deberá revisar y adelantar las respectivas acciones de limpieza a cualquier maquinaria y objeto, que hayan estado en zonas donde exista el retamo.

Con el fin de evitar la dispersión no intencional, deberán diseñarse campañas divulgativas que indiquen las medidas que se deben adoptar para así lograr evitar la dispersión de semillas por medio ropa, calzado, en máquina, herramienta, animal doméstico.

\section{ERRADICACIÓN}

-Posteriormente a la eliminación del material vegetal extraído, se deberá realizar un control periódico a los rebrotes. Teniendo en cuenta que la mayoría de las semillas de las especies de Retamo, se encuentran entre los 0 y $12 \mathrm{~cm}$ de profundidad.

-Una estrategia útil para disminuir el banco de semillas es la remoción del suelo con azadón o rastrillo el cual solo se deberá utilizar en la primera intervención de remoción, y así exponer las semillas para que germinen y posteriormente eliminar las plántulas.

\section{MANEJO DE RESIDUOS VEGETALES POST CORTE}

-Para realizar la transformación del material cortado (trituración), se deberá separar las ramas de los troncos, para así facilitar su corte a través de una máquina trituradora.

-El suelo del área donde se ubique esta máquina, debe estar cubierto por plástico grueso mayor a calibre seis (6), para así evitar la dispersión de semillas; o mediante lonas o algún material resistente de difícil rompimiento que no permita que las semillas lo traspasen y la acumulación de semillas de retamo en el área de procesamiento.

-El embalaje de todas las piezas debe hacerse en lonas de polipropileno que no presenten filtraciones y estar debidamente aseguradas, para que las semillas no se dispersen durante el transporte.

El transporte de la especie no requerirá se salvoconducto único de movilización, debido a que esta especie no tiene distribución natural en Colombia. Para su transporte, se deberá consignar en las bitácoras de movilización de las autoridades ambientales respectivas, la zona donde se hizo la recolección y el lugar de la disposición final.

-El aislamiento y encerramientos son actividades que se realizan con el objetivo de disminuir y concentrar la dispersión de semillas del retamo, las actividades de eliminación, transformación y destrucción; así como para evitar el ingreso de animales y personal no autorizado que pueda facilitar la dispersión de las semillas fuera del área o puede favorecer incendios.

TERCERA FASE - EXPERIMENTAL: Se realiza el proceso de obtención de azucares vía hidrolisis acida.

Se utilizó retamo espinoso (Ulex europeaus) previamente molido, el cual fue llevado a tratamiento de 
reducción de tamaño de $1 \mathrm{~mm}$ por medio de tamices.

La hidrólisis ácida se llevó a cabo en:

-Matraces Erlenmeyer de $250 \mathrm{~mL}$, los cuales contenían $5 \mathrm{~g}$ de Retamo espinoso y $100 \mathrm{~mL}$ de $\mathrm{H}_{2} \mathrm{SO}_{4}$ en concentración de $0.1,0.3,0.5 \mathrm{~N}$.

-Los matraces se mantuvieron a una temperatura de $30^{\circ} \mathrm{Cy} 150 \mathrm{rpm}$ en un agitador orbital (IKA KS $4000 \mathrm{i}$ control).

Determinación de azúcares reductores

La cuantificación de azucares reductores en los hidrolizados se determinó utilizando el método fotocolorimétrico DNS (Dobois et al., 1956). Basado en la reducción de este reactivo, lo cual hace referencia al cambio de color durante su reacción.

Para la preparación del reactivo: 


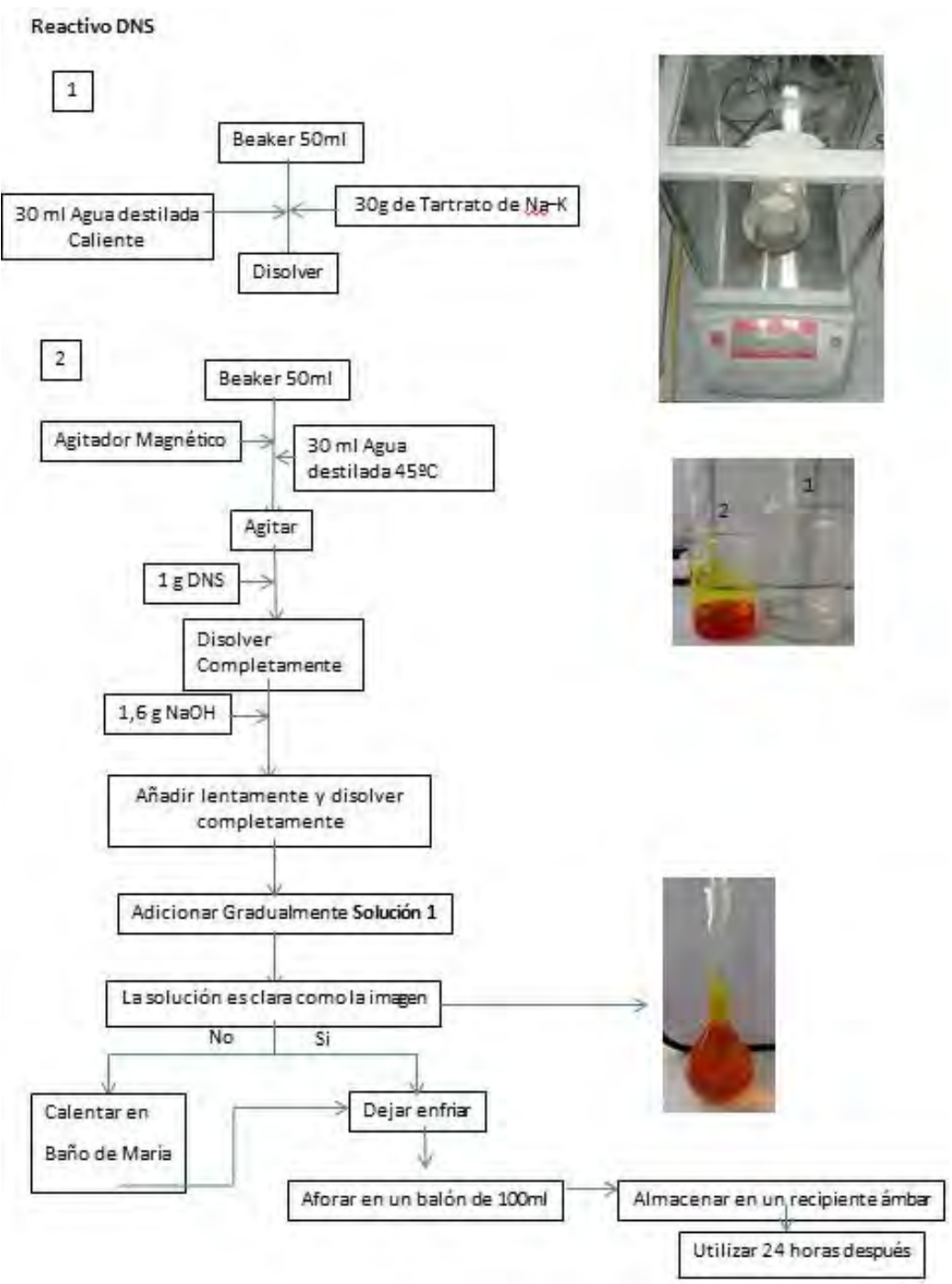

Patrón de glucosa

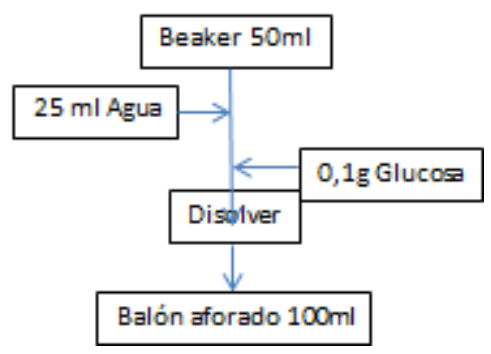

ENCUENTRO CON SEMILLEROS, APORTES Y REFLEXIONES

Volumen 1 No 1 (2019) ISSN 2711-4414 

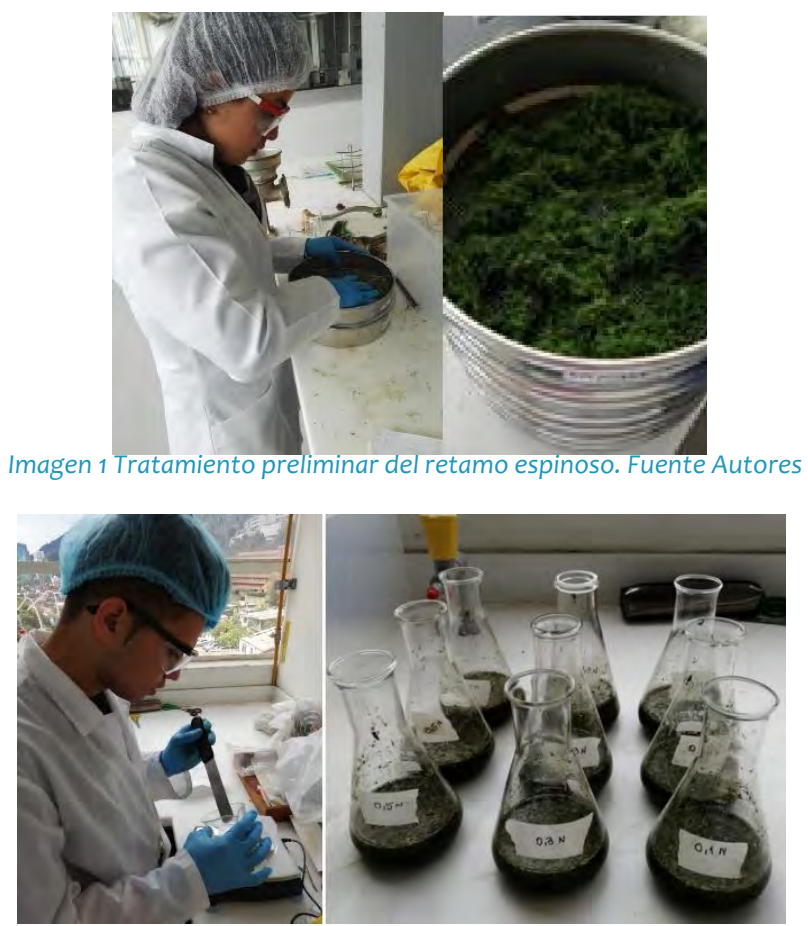

Imagen 2 Proceso de hidrolisis ácida. Fuente Autores

\section{Curva de Calibración}

Para la curva de calibración del método, colocar en una gradilla 10 tubos y marcarlos según la siguiente numeración, adicionando las siguientes cantidades de sustancias que corresponda y seguir los pasos establecidos:

\begin{tabular}{|c|c|c|c|c|c|c|}
\hline Tubo & $\begin{array}{c}\text { Concentración } \\
(\mathrm{mg} / \mathrm{mL})\end{array}$ & $\begin{array}{c}\text { Patrón de } \\
\text { Glucosa }(\mu \mathrm{l})\end{array}$ & $\begin{array}{c}\text { Agua } \\
(\mu \mathrm{l})\end{array}$ & $\begin{array}{l}\text { DNS } \\
(\mu \mathrm{l})\end{array}$ & \multirow{12}{*}{$\begin{array}{l}\text { Termoreactor } \\
5 \mathrm{~min} 100^{\circ} \mathrm{C}\end{array}$} & $\begin{array}{c}\text { Agua } \\
(\mu \mathrm{l})\end{array}$ \\
\hline Blanco & 0 & 0 & 1000 & \multirow{11}{*}{1000} & & \multirow{11}{*}{8000} \\
\hline 1 & 0,1 & 100 & 900 & & & \\
\hline 2 & 0,2 & 200 & 800 & & & \\
\hline 3 & 0,3 & 300 & 700 & & & \\
\hline 4 & 0,4 & 400 & 600 & & & \\
\hline 5 & 0,5 & 500 & 500 & & & \\
\hline 6 & 0,6 & 600 & 400 & & & \\
\hline 7 & 0,7 & 700 & 300 & & & \\
\hline 8 & 0,8 & 800 & 200 & & & \\
\hline 9 & 0,9 & 900 & 100 & & & \\
\hline 10 & 1 & 1000 & 0 & & & \\
\hline
\end{tabular}

Tabla 1. Diluciones del patrón de glucosa. Fuente 
Resultados

\section{Curva de Calibración}

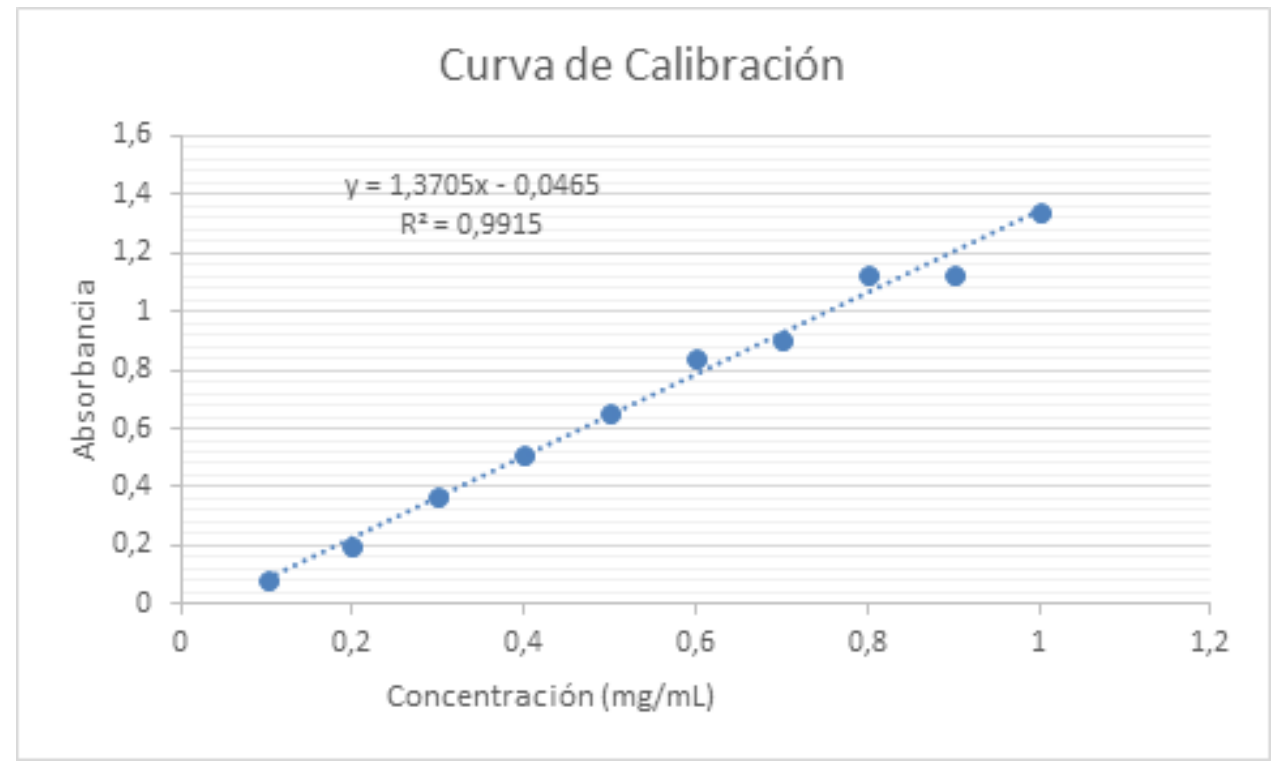

Grafica 1. Curva de calibración. Fuente Autores

Teniendo los valores de absorbancia de muestras (Tabla 1) y la ecuación de la pendiente de la curva de calibración que se encuentra en la Gráfica 1. Curva de calibración, se calcula la cantidad de azúcares presentes en las muestras como se muestra a continuación:

Teniendo en cuenta la curva de calibración se calcularon las concentraciones a partir de:

Ecuación curva de calibración

$Y=1,3705 X-0,0465$

$X=Y+0,0465 / 1,3705$

$y=$ Absorbancia

$X=$ Concentración de glucosa $\mathrm{mg} / \mathrm{mL}$

\begin{tabular}{|c|c|c|c|r|r|}
\hline $\begin{array}{c}\text { Concentración de } \\
\text { H2SO4 }\end{array}$ & Muestra & Absorbancia & $\begin{array}{c}\text { Concentración(mg/ } \\
\mathrm{mL} \text { de azúcares) }\end{array}$ & $\begin{array}{c}\text { Concentración en mg/L } \\
\text { de AR }\end{array}$ & $\begin{array}{l}\text { Concentración } \\
\text { media mg/L AR }\end{array}$ \\
\hline \multirow{2}{*}{$0,1 \mathrm{~N}$} & 1 & 3,852 & 2,845 & 2845 & \multirow{2}{*}{2801,66667} \\
\cline { 2 - 5 }
\end{tabular}

ENCUENTRO CON SEMILLEROS, APORTES Y REFLEXIONES

Volumen 1 No 1 (2019) ISSN 2711-4414 


\begin{tabular}{|c|c|c|c|c|c|}
\hline & 3 & 3,756 & 2,775 & 2775 & \\
\hline \multirow{3}{*}{$0,3 \mathrm{~N}$} & 1 & 2,011 & 1,501 & 1501 & \multirow{3}{*}{1450} \\
\hline & 2 & 2,012 & 1,502 & 1502 & \\
\hline & 3 & 1,8 & 1,347 & 1347 & \\
\hline \multirow{3}{*}{$0,5 \mathrm{~N}$} & 1 & 0,01 & 0,041 & 41 & \multirow{3}{*}{82,6666667} \\
\hline & 2 & 0,163 & 0,153 & 153 & \\
\hline & 3 & 0,028 & 0,054 & 54 & \\
\hline
\end{tabular}

Tabla 1. Concentración y absorbancia de muestras. Fuente Autores

Por último, se relaciona la cantidad de azúcar reductor producida por g de residuo seco de retamo espinoso, teniendo en cuenta la humedad de cada biomasa como se muestra en la siguiente ecuación:

$\operatorname{mg} A R$

$m g$

Volumen adicionado $(L)$

peso inicial - \%hmedad 100

$$
\left.\frac{\text { gde residuo } \sec o}{\text { dencentracion real }(}{ }_{L}\right) *
$$

En la tabla 2 se muestran los resultados para cada tratamiento teniendo en cuenta que el contenido de humedad

para el retamo espinoso fue de $64.7 \%$

\begin{tabular}{|c|c|c|c|c|}
\hline $\begin{array}{c}\text { Cantidad de Retamo } \\
\text { (g) }\end{array}$ & $\begin{array}{c}\text { volumen } \\
(\mathrm{L})\end{array}$ & $\begin{array}{c}\text { Peso seco mg AR/g de } \\
\text { residuo seco }\end{array}$ & $\begin{array}{c}\text { Cantidad de azúcares (mg } \\
\text { AR/g de residuo seco) }\end{array}$ & $\begin{array}{c}\text { Porcentaje de azucares } \\
\text { reductores respecto a 1 g de } \\
\text { residuo seco }\end{array}$ \\
\hline 5 & 0,1 & 1,765 & 158,7 & 15,9 \\
\hline 5 & 0,1 & 1,765 & 82,2 & 8,2 \\
\hline 5 & & & & 0,5 \\
\hline
\end{tabular}

Tabla 2. Contenido de azúcar obtenido por gramo de residuo seco. Fuente Autores

\section{Discusión y Conclusión}

Según la tabla 2 La mayor liberación de azúcares reductores se dio para el tratamiento del residuo por hidrólisis ácida a una concentración de $0.1 \mathrm{~N}$ obteniendo un valor de 158,7 mg AR/g de residuo seco y 82,2 mg AR/ g de residuo seco para la concentración de ácido al 0,3 N, ambos con una relación 1:10 peso a líquido.

Se evidencia en los resultados que las mejores condiciones de hidrólisis ácida para el retamo espinoso fueron de ácido sulfúrico a una concentración de $0.1 \mathrm{~N}$, relación 1:10 peso: líquido, en un tiempo de 24 horas, agitación de $150 \mathrm{rpm}$ y una temperatura de $30{ }^{\circ} \mathrm{C}$. Donde en esta concentración de ácido sulfúrico se obtuvo una conversión en azúcares reductores del 16\%, respecto a las otras dos concentraciones 0,3 N y 0,5 N de ácido, la conversión fue de $8 \%$ y $0,5 \%$ respectivamente, tomando como base el residuo seco utilizado. Se puede concluir

\section{ENCUENTRO CON SEMILLEROS, APORTES Y REFLEXIONES Volumen 1 No 1 (2019) ISSN 2711-4414}


que la humedad juega un papel importante en la liberación de azúcares reductores de la biomasa.

Para el retamo espinoso, se dio mejor liberación de azúcares con la mínima concentración de ácido que se utilizó, por lo que se puede decir que el rango de concentraciones para la hidrólisis está entre $0.005 \mathrm{~N}$ y $0.1 \mathrm{~N}$ en concentraciones del ácido para el retamo espinoso.

Según los resultados obtenidos es necesario seguir haciendo estudios en donde se varíen las condiciones de hidrólisis, para así obtener las mejores condiciones para el escalamiento a un nivel piloto de producción de estos azúcares y por ende su fermentación para convertirse en Biocombustible de segunda generación.

\section{Referencias bibliográficas}

Aguilar, N (2010). MODELO CINÉTICO DE LA HIDRÓLISIS DEL RESIDUO DE COSECHA CAÑERO, Ciencia e Ingeniería Chandler, C., Villalobos, N., González, E., Arenas, E., Mármol, Z., Ríos, J., \& Aiello Mazzarri, C. (2013). Hidrólisis ácida diluida en dos etapas de bagazo de caña de azúcar para la producción de azúcares fermentables. Retrieved from http://hemeroteca.lasalle.edu.co/login?url=http://search.ebscohost.com/login.aspx?direct=true\&AuthTyp e=ip, ur l,uid\&db=edsbas\&AN=edsbas.5F7DAD59\&lang=es\&site=eds-live

Chapín, G. A., Sarrouh, B. F., De la Prida, J. J., \& Suarez, E. G. (2006). Estudio De La Hidrólisis Del Bagazo Con Ácido Sulfúrico Concentrado Para La Obtención De Etanol Y Análisis Técnico Económico De Dicho Proceso. Revista Cubana de Química, 18(1), 95. Retrieved from http://hemeroteca.lasalle.edu.co/login?url=http://search.ebscohost.com/login.aspx?direct=true\&AuthTyp e=ip,ur l,uid\&db=zbh\&AN=33321886\&lang=es\&site=eds-live

Domínguez Domínguez, M., Álvarez Castillo, A., Castrejón Rosales, T., Granados Baeza, M., Hernández Campos , F., Alcalá Octaviano , V., \& Tapia Picazo, J. C. (2011). Estudio de la cinética del hidrólisis ácida del bagazo de caña de azúcar sin pretratamiento para la obtención de azúcares reductores. Revista Iberoamericana de Polímeros, 153-159.

Ecofondo. (22 de 02 de 2019). Obtenido de http://ecofondo.org.co/articulo.php?id=71

Estrada, C (2010). Universidad Nacional Autonoma de Mexico Facultad de Quimica Quimica de Alimentos I. Celulosa, (Mcc)

Giraldo, L. F. M., Correa, H. A. M., Gutiérrez, J. E. B., \& Castaño, C. E. C. (2007). Aprovechamiento del residuo agroindustrial del mango común (Mangifera indica L.) en la obtención de azúcares fermentables. Ingeniería y Ciencia, 3(6), 41-62.

González García, Y., Meza Contreras, J. C., Anzaldo Hernández, J., \& Sanjuán Dueñas, R. (2015). Obtención de azúcares fermentables desde residuos de cartón para cultivar levaduras de interés biotecnológico / Procurement of fermentable sugars from cardboard waste for the cultivation of yeasts for biotechnological use. Revista Mexicana de Ciencias Forestales, (28), 88.

Giraldo, L. F. M., Correa, H. A. M., Gutiérrez, J. E. B., \& Castaño, C. E. C. (2007). Aprovechamiento del residuo agroindustrial del mango común (Mangifera indica L.) en la obtención de azúcares fermentables. Ingeniería y Ciencia, 3(6), 41-62.

Hackenberg, N. (2008). Biocombustibles de segunda generacion Revista Virtual REDESMA, 49-55.

Hackenberg, N (2008) Biocombustibles de segunda generación. Instituto Ecuatoriano de Normalización (2012). Norma técnica ecuatoriana

Herrera, P., Hernández, E., Morales, M., \& Ortiz, I. (2007). Hidrólisis ácida de biomasa lignocelulósica y algal para la obtención de azúcares fermentables para la producción de etanol. XIV Congreso nacional de biotecnología y bioingeniería.

JIMÉNEZ ISLAS, D., ABREU CORONA, A., LÓPEZ Y LÓPEZ, V. E., TELLEZ JURADO, A., \& GRACIDA RODRÍGUEZ, J. 
N. (2012). Obtención de azúcares fermentables mediante hidrólisis ácida de Beta vulgaris L. / Fermentable sugar production throug acid hydrolysis of Beta vulgaris L. Revista Internacional de Contaminación Ambiental, (2), 151.

Tejada Benítez, L., Tejada Tovar, C., Villabona Ortiz, Ángel, Tarón Dunoyer, A., Alvear, M., Castillo, C., Henao, D., Marimón, W., \& Madariaga, N. (2010). Producción de bioetanol a partir de la fermentación alcohólica de jarabes glucosados derivados de cáscaras de naranja y piña. Revista Educación En Ingeniería, 5(10), 120-125. https://doi.org/10.26507/rei.v5n10.104 https://educacioneningenieria.org/index.php/edi/article/view/104

Tejeda L, Alvear M, Henao D (2014). Evaluación del potencial de las cáscaras de frutas en la obtención de bioetanol. Tomado de:file://C:/Users/Usuario/Downloads/323244-107262-1-PB.pdf

SÁNCHEZ-OROZCO, Raymundo \& VÁZQUEZ VELÁZQUEZ, José. Hidrólisis de residuos de naranja para la obtención de carbohidratos fermentables. Revista de Investigación y Desarrollo.2017, 3-9: 63-70.

Neogranadina, Vol. 20-2, pp. 5-18. Bogotá. ISSN 0124-8170 